\title{
The American Joint Committee on Cancer 8th edition staging system for the pancreatic ductal adenocarcinoma: is it better than the 7 th edition?
}

\author{
Dong Woo Shin, Jaihwan Kim \\ Division of Gastroenterology, Department of Internal Medicine, Seoul National University College of Medicine, Seoul National University Bundang \\ Hospital, Seongnam, Korea \\ Correspondence to: Jaihwan Kim, MD. Department of Internal Medicine, Seoul National University Bundang Hospital 82, Gumi-ro 173 beon-gil, \\ Bundang-gu, Seongnam, Gyeonggi-do, 463-707, South Korea. Email: drjaihwan@snubh.org. \\ Comment on: Shi S, Hua J, Liang C, et al. Proposed Modification of the 8th Edition of the AJCC Staging System for Pancreatic Ductal \\ Adenocarcinoma. Ann Surg 2019;269:944-50.
}

Submitted Aug 05, 2019. Accepted for publication Aug 12, 2019.

doi: 10.21037/hbsn.2019.08.06

View this article at: http://dx.doi.org/10.21037/hbsn.2019.08.06

Pancreatic ductal adenocarcinoma (PDA) is the fourth leading cause of cancer-related death and has a poor prognosis with a 5-year survival rate of $9 \%$. In 2019, 56,770 new PDAs and 45,750 deaths are projected to occur in the United States (1). Because of the dismal prognosis, it is important to predict the course of the disease precisely. The TNM staging system is a widely used not only for predicting prognosis but also for collecting and exchanging cancer information. The American Joint Committee on Cancer (AJCC) Cancer Staging Manual, 8th edition was introduced in October 2016 and there were many changes in the staging of PDA compared with the previous one. Of the several controversies related with the previous edition, the most important one was the reproducibility of the T-category. In the 7 th edition, there were discrepancies in opinion among pathologists about pT3 (extension beyond the pancreas). Because the pancreas does not have a capsule, it is difficult to determine if there is an extra-pancreatic extension in the presence of inflammation or desmoplasia. Therefore, in the revised staging system, T-category was classified by size, regardless of the extrapancreatic invasion: $\mathrm{pT} 1, \leq 2 \mathrm{~cm}$ in maximal diameter; $\mathrm{pT}$, $>2 \mathrm{~cm}$ but $\leq 4 \mathrm{~cm} ; \mathrm{pT} 3,>4 \mathrm{~cm}$; pT4, locally unresectable due to involvement of major arteries (the celiac axis or the superior mesenteric artery). Another controversy in the 7th edition was that the $\mathrm{N}$-category is simply divided into $\mathrm{pN} 0$ and $\mathrm{pN} 1$. It has been suggested that the $\mathrm{N}$-category should be subdivided because the number of metastatic lymph nodes (LNs) affects prognosis (2). Consequently, the previous node- positive $\mathrm{pN} 1$ category has been subdivided into $\mathrm{pN} 1$ (1-3 metastatic LNs) and $\mathrm{pN} 2$ (4 or more metastatic LNs) in the revised edition and $\mathrm{pN} 2$ was defined as stage III regardless of tumor size.

In order to determine whether the AJCC 8th edition staging system predicts the prognosis appropriately, we need to consider the following two questions: (I) Do new Tand $\mathrm{N}$-category adequately stratify the prognosis? (II) Do the TNM stage and its substages show higher prognostic accuracy than the previous edition?

The answer to the first question was reported in several large-scale studies (2-5). Recently, a multi-institutional study (3) and a large-scale study based on the Surveillance, Epidemiology and End Results (SEER) database (4) evaluated the AJCC 8th edition staging system for PDA. Allen et al. showed that the revised staging system is statistically valid in a study using prospective databases at three large US centers (3). The shift to new T-category based on size alone showed reproducible results among pathologists in three institutions. Kamarajah et al. suggested that the prognosis of patients with PDA could be divided by the newly proposed $\mathrm{N}$-category (4). Liu et al. also reported that the newly proposed $\mathrm{T}$ - and $\mathrm{N}$-category could be used as a prognostic factor in a study of 18,450 patients with PDA in the SEER database (5). In conclusion, the Tand $\mathrm{N}$-category of the AJCC 8th edition can adequately distinguish the prognosis of patients with PDA.

Regarding the second question, several studies have 
Table 1 The definition of TNM category in American Joint Committee on Cancer (AJCC) 7th and 8th edition staging system and the modified 8th staging system

\begin{tabular}{|c|c|c|}
\hline TNM category & Stage & Modified stage (by Shi et al.) \\
\hline \multicolumn{3}{|l|}{ AJCC 8th edition } \\
\hline $\mathrm{T} 1$, Maximum tumor diameter $\leq 2 \mathrm{~cm}$ & Stage IA, T1 NO MO & Stage IA, T1 NO M0 \\
\hline T3, Maximum tumor diameter $>4 \mathrm{~cm}$ & Stage IIA, T3 NO MO & $\begin{array}{l}\text { Stage IIA, T3 N0 M0, T2 N1 M0, T1 } \\
\text { N2 M0 }\end{array}$ \\
\hline N0, No regional LN metastasis & Stage III, $\mathrm{T}_{\text {any }} \mathrm{N} 2 \mathrm{M} 0, \mathrm{~T} 4 \mathrm{~N}_{\text {any }} \mathrm{M} 0$ & Stage IIIA, T3 N2 M0 \\
\hline N1, Metastasis in $1-3$ regional LNs & - & Stage IIIB, T4 $\mathrm{N}_{\text {any }} \mathrm{M} 0$ \\
\hline N2, Metastasis in $\geq 4$ regional LNs & Stage IV, $\mathrm{T}_{\text {any }} \mathrm{N}_{\text {any }} \mathrm{M} 1$ & Stage IV, $\mathrm{T}_{\text {any }} \mathrm{N}_{\text {any }} \mathrm{M} 1$ \\
\hline \multicolumn{3}{|l|}{ M0, No distant metastasis } \\
\hline T2, Tumor limited to the pancreas, $>2 \mathrm{~cm}$ in maximal diameter & Stage IB, T2 NO MO & \\
\hline $\begin{array}{l}\text { T3, Tumor extends beyond the pancreas (without involvement of the } \\
\text { CA or the SMA) }\end{array}$ & Stage IIA, T3 NO MO & \\
\hline T4, Tumor involves the CA or the SMA (unresectable primary tumor) & Stage IIB, T1-3 N1 M0 & \\
\hline N0, No regional LN metastasis & Stage III, T4 $\mathrm{N}_{\text {any }} \mathrm{MO}$ & \\
\hline N1, Regional LN metastasis & Stage IV, $\mathrm{T}_{\text {any }} \mathrm{N}_{\text {any }} \mathrm{M} 1$ & \\
\hline \multicolumn{3}{|l|}{ Mo, No distant metastasis } \\
\hline M1, Distant metastasis & & \\
\hline
\end{tabular}

AJCC, American Joint Committee on Cancer; CA, celiac axis; SMA, superior mesenteric artery; LN, lymph node.

suggested that the new TNM stage can adequately classify the prognosis $(3,4,6)$. In a multi-institutional study of 2,318 patients with PDA who underwent R0 resection, Allen et al. showed that a new staging system evenly stratified the stages without loss of prognostic accuracy (3). In a study of 8,960 patients who underwent surgical resection for a nonmetastatic PDA using the SEER database, the concordance index, which represents discriminatory power, is higher in the revised staging system than in previous one (4). In another study of 701 resected PDAs from South Korea, it was also found that the AJCC 8th edition staging system appropriately stratified the prognosis (6). However, Shi et al. suggested the modified staging system according to the survival of patients with each substages. They analyzed data of 45,856 patients with PDA in the SEER database (20042014) and 3,166 patients with PDA in the Fudan University Shanghai Cancer Center (FUSCC) database (20052015) (7). They calculated the median overall survival (OS) according to the substages to assess the accuracy and appropriateness of the AJCC 8th edition staging system and found that there was a significant difference in median OS according to the substages. The median OS of the three substages belonging to stage IIB was different according to T stages (T1N1M0 23.0, T2N1M0 19.0, and T3N1M0 16.0 months; $\mathrm{P}<0.001)$. Furthermore, stage IIB had significantly longer median OS than stage IIA (T1N1M0, 23.0 months and T3N0M0, 20.0 months; $\mathrm{P}<0.001)$. Also, stage III had significantly longer survival than stage IIB (T1N2M0, 20.0 months vs. T3N1M0, 16.0 months; $\mathrm{P}<0.001)$. Because the prognosis of the two subgroups of stage III was different, they suggested separation of stage III into IIIA and IIIB (T3N2M0, 13.0 months and $\mathrm{T}_{4} \mathrm{~N}_{\text {any }} \mathrm{M} 0,10.0$ months, respectively). Consequently, they proposed a modified staging system regrouping substages, while maintaining $\mathrm{T}$ - and $\mathrm{N}$-categories (Table 1). The staging system newly proposed by them showed a superior 
concordance index over the existing AJCC 8th edition staging system (0.637 vs. 0.620). Although this study is retrospective design, it is necessary to validate this result in future studies, because the data used in this study were extracted from a large number of patients based on two different databases.

In summary, the AJCC 8th edition staging system was more reproducible and enabled finer stratification of the prognosis than the previous edition. A new definition of subdivision of $\mathrm{T}$ - and $\mathrm{N}$-category has made the staging system of PDA more accurate. However, there is still a room for improvement. The modified staging system proposed in this validation study could be considered for adoption in the next edition of the AJCC staging system.

\section{Acknowledgments}

None.

\section{Footnote}

Conflicts of Interest: The authors have no conflicts of interest to declare.

Ethical Statement: The authors are accountable for all aspects of the work in ensuring that questions related to the accuracy or integrity of any part of the work are appropriately investigated and resolved.

Cite this article as: Shin DW, Kim J. The American Joint Committee on Cancer 8th edition staging system for the pancreatic ductal adenocarcinoma: is it better than the 7 th edition? Hepatobiliary Surg Nutr 2020;9(1):98-100. doi: 10.21037/hbsn.2019.08.06

\section{References}

1. Siegel RL, Miller KD, Jemal A. Cancer statistics, 2019. CA Cancer J Clin 2019;69:7-34.

2. Malleo G, Maggino L, Capelli P, et al. Reappraisal of Nodal Staging and Study of Lymph Node Station Involvement in Pancreaticoduodenectomy with the Standard International Study Group of Pancreatic Surgery Definition of Lymphadenectomy for Cancer. J Am Coll Surg 2015;221:367-79.e4.

3. Allen PJ, Kuk D, Castillo CF, et al. Multi-institutional Validation Study of the American Joint Commission on Cancer (8th Edition) Changes for T and N Staging in Patients With Pancreatic Adenocarcinoma. Ann Surg 2017;265:185-91.

4. Kamarajah SK, Burns WR, Frankel TL, et al. Validation of the American Joint Commission on Cancer (AJCC) 8th Edition Staging System for Patients with Pancreatic Adenocarcinoma: A Surveillance, Epidemiology and End Results (SEER) Analysis. Ann Surg Oncol 2017;24:2023-30.

5. Liu C, Cheng H, Jin K, et al. Application of the Eighth Edition of the American Joint Committee on Cancer Staging for Pancreatic Adenocarcinoma. Pancreas 2018;47:742-7.

6. Shin DW, Lee JC, Kim J, et al. Validation of the American Joint Committee on Cancer 8th edition staging system for the pancreatic ductal adenocarcinoma. Eur J Surg Oncol 2019. [Epub ahead of print].

7. Shi S, Hua J, Liang C, et al. Proposed Modification of the 8th Edition of the AJCC Staging System for Pancreatic Ductal Adenocarcinoma. Ann Surg 2019;269:944-50. 